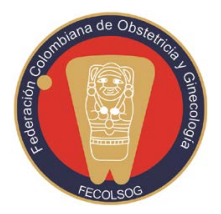

Revista Colombiana de Obstetricia y Ginecología Vol. 67 No. 4 • Octubre-Diciembre 2016 • (271-277)

\title{
A PROPÓSITO DE UNA NUEVA CLASIFICACIÓN DEL EMBARAZO A TÉRMINO. RESULTADOS NEONATALES EN UNA CLÍNICA DE TERCER NIVEL DE ATENCIÓN EN CALI, COLOMBIA. UN ESTUDIO DE CORTE TRANSVERSAL, 2013
}

\section{In view of a new classification of term pregnancy Neonatal outcomes in a level IIII clinic in Cali, Colombia A cross-sectional study, 2013}

Rodrigo Cifuentes-Borrero, $M D, \mathrm{PhD}^{1}$; Mauricio Hernández-Carrillo, $\mathrm{MSc}^{2}$; Ana María Toro-Cifuentes, $M D^{3}$; Viviana R. Franco-Torres, $M D^{3}$; Ángela M. Cubides-Munévar, $\mathrm{MSc}^{4}$; Ingrid J. Duarte-González, $\mathrm{MD}^{3}$

Recibido: octubre 26/15 - Aceptado: octubre 26/16

\section{RESUMEN}

Objetivo: aplicar la clasificación del parto a término del American College of Obstetricians and Gynaecologists (ACOG); describir la frecuencia de las diferentes categorías y hacer un análisis exploratorio de los resultados neonatales entre los grupos.

Materiales y métodos: estudio de corte transversal. Se incluyeron gestantes consideradas inicialmente sanas, con embarazo a término definido según última menstruación confiable y ecografía en la primera mitad de la gestación, de una clínica privada de tercer nivel, de Cali (Colombia), año 2013. Los partos se clasificaron como parto a término temprano, a término y a término tardío. Se describen las características socio-

1 Coordinador de posgrado en Ginecología y Obstetricia, Universidad Libre de Cali; director Grupo interinstitucional de Obstetricia y Ginecología (GIGyO), Cali (Colombia).rcifuentesmd@gmail.com

2 Estadístico y Magíster en Epidemiología; Investigador Grupo GISAP, Universidad Santiago de Cali, Cali (Colombia).mauriciohc@gmail.com

3 Médico residente en Ginecología y Obstetricia, Universidad Libre de Cali, Cali (Colombia).

4 Magíster en Epidemiología; directora Grupo GISAP, Universidad Santiago de Cali, Cali (Colombia). angelacubides.epi@gmail.com demográficas maternas y los resultados perinatales, así como la frecuencia de cada categoría; la comparación de grupos se estableció a través de análisis de varianza (ANOVA), Kruskal-Wallis o chi cuadrado.

Resultados: de 502 nacimientos, fueron clasificados como embarazos a término temprano 200 (39,8\%), a término completo 254 (50,6\%) y tardío 48 (9,6\%). Hubo mayor frecuencia de aseguramiento contributivo de la madre y embarazos de alto riesgo en el grupo a término temprano. Se observaron diferencias estadísticamente significativas en bajo peso al nacer y peso promedio del neonato en el grupo a término temprano, y mayor asfixia neonatal en el grupo a término tardío. No hubo diferencias en cuanto a síndrome de dificultad respiratoria y tiempo de hospitalización.

Conclusión: la nueva clasificación de embarazo a término de la ACOG es factible de aplicar. La frecuencia de parto a término temprano es alta en Colombia. No se evidenciaron diferencias significativas en los resultados neonatales entre los tres grupos excepto en la asfixia neonatal, que fue mayor en el grupo a término tardío. 
Palabras clave: recién nacido, trabajo de parto prematuro, parto, nacimiento prematuro, nacimiento a término, embarazo.

\section{ABSTRACT}

Objective: To apply the classification of term delivery of the American College of Obstetricians and Gynaecologists, describe the frequency of the various categories, and explore neonatal outcomes among groups.

Materials and methods: A cross-sectional study including pregnant women considered initially healthy with a term pregnancy defined on the basis of the last reliable menstruation and ultrasound performed in the first half of the gestation period in a private, Level III clinic, in Cali, Colombia, in 2013. Deliveries were classified as early term, full term and late term. Social and demographic characteristics of the mothers and perinatal outcomes are described. The frequency of each category is described and group comparisons are performed using the variance analysis (ANOVA), and the Kruskal-Wallis or Chi-square test.

Results: Of 502 births, 200 (39.8\%) were classified as early term, $354(50.6 \%)$ as full term, and 48 $(9.6 \%)$ as late term. There was a higher frequency of contributive insurance coverage and high risk pregnancies in the early term group. Statistically significant differences were observed in terms of low birth weight and average neonatal weight in the early term group, whereas neonatal asphyxia was higher in the late term group. There were no differences in terms of respiratory distress syndrome or length of stay.

Conclusion: It is feasible to apply the new ACOG term pregnancy classification. The frequency of early term delivery is high in Colombia. There was no evidence of significant differences in neonatal outcomes between the three groups except for higher neonatal asphyxia in the late term group.

Key words: Newborn, premature labour, premature birth, term delivery, pregnancy.

\section{INTRODUCCIÓN}

El embarazo a término es aquel que termina entre tres semanas antes y dos después de la fecha estimada del parto (1). Esta definición incluye un periodo de 6 semanas en la época de mayor crecimiento fetal, y cuando se considera que están maduros los sistemas para un recién nacido sano (2). Sin embargo, esta definición ha sido cuestionada por estudios que han mostrado una mayor frecuencia de complicaciones neonatales en los embarazos de 37,0 a 38,6 semanas que en aquellos de 39 a 41 semanas (3, 4). A partir de estas observaciones, el American College of Obstetricians and Gynaecologists (ACOG) propone una nueva clasificación del embarazo a término, en la cual los embarazos entre 37 semanas 0/7 días y 38 semanas 6/7 días se denominan embarazos a término temprano; los que tienen entre 39 semanas $0 / 7$ días y 40 semanas $6 / 7$ días son embarazos a término; los de 41 semanas 0/7 días y 41 semanas 6/7 días, término tardío, y los de 42 semanas 0 días y más, postérmino (5). Bajo esta clasificación, el $18 \%$ de todos los nacimientos, y una tercera parte de las finalizaciones electivas de embarazos corresponderían a nacimientos a término temprano (6). Esta clasificación, sin embargo, es motivo de controversia ya que su implementación no ha tenido el impacto esperado en términos de ingresos a las unidades de cuidados intensivos neonatales y un posible incremento de macrosomía y muerte fetal (7).

La información sobre los resultados neonatales de los fetos a término según la clasificación internacional de enfermedades es aún escasa, y no se ha hecho localmente una evaluación según la clasificación propuesta por ACOG. Por tanto, el objetivo del presente estudio es hacer una descripción de la frecuencia de los partos a término en cada una de las subcategorías propuestas y hacer un análisis exploratorio sobre una posible asociación entre los fetos a término temprano y malos resultados perinatales en nuestra población. 


\section{MATERIALES Y MÉTODOS}

Se realizó un estudio descriptivo de corte transversal. Se incluyeron gestantes consideradas inicialmente sanas, con embarazo a término definido según última menstruación confiable y ecografía en la primera mitad de la gestación, atendidas durante el año 2013 en una clínica privada de tercer nivel, de Cali (Colombia), a la cual acuden pacientes obstétricas de bajo y alto riesgo, pertenecientes al régimen subsidiado por el Estado y del régimen contributivo, según la clasificación del sistema de seguridad social en salud de Colombia. Se realizó muestreo consecutivo estricto. Como muestra se tomó el total de registros disponibles en el periodo comprendido entre el 01 de enero de 2013 y el 31 de diciembre de 2013.

Procedimiento. A partir de la historia clínica sistematizada, se utilizó el total de registros disponibles en el periodo mencionado. La información contenida en la historia clínica fue revisada previa a su ingreso en la base de datos realizada para consolidar las variables de interés del estudio.

Se midieron las siguientes variables de la madre: edad, afiliación de la madre al sistema general de seguridad social en Colombia, número de controles prenatales, días de hospitalización, riesgo del embarazo (alto o bajo), diagnóstico materno al ingreso y relacionado con el ingreso del feto. En cuanto al recién nacido: peso en gramos del menor al nacimiento, bajo peso al nacer (definido como peso por debajo de $2500 \mathrm{~g}$ ) talla del nacido vivo, presencia de hipoglicemia, macrosomía o ictericia. Apgar al minuto después del nacimiento, Apgar a 1 y 5 minutos después del nacimiento,, dificultad respiratoria, asfixia perinatal y aspiración de meconio. Infecciones. Meconio, sepsis o corioamnionitis. Adicionalmente, se midió el número de días de hospitalización del recién nacido.

Análisis estadístico. Los datos fueron analizados por medio del programa R 3.2.0. Se realizó análisis descriptivo de los datos; se presentan las medias con su respectiva desviación estándar, o medianas con rango intercuartílico (RIC) para las variables cuantitativas, y frecuencias absolutas y relativas para las variables cualitativas. Se evaluó el ajuste de los datos a la distribución normal mediante la prueba de Shapiro-Wilk. La comparación de grupos (a término temprano de 37 a 38,6 semanas, a término completo de 39 a 40,6 semanas y a término tardío de 41 a 41,6 semanas) se estableció a través de: análisis de varianza (ANOVA) en los casos donde se cumplió el supuesto de normalidad y se compararon promedios, Kruskal-Wallis para comparación de medianas, Prueba $\mathrm{Z}$ de proporciones de $\mathrm{k}$ muestras y prueba chi cuadrado según correspondió de acuerdo con los resultados de las pruebas de normalidad.

Aspectos éticos. Esta investigación contó con la aprobación del comité de ética de la Clínica Universitaria Rafael Uribe Uribe y de la Universidad Libre, Cali.

\section{RESULTADOS}

De 502 nacimientos fueron clasificados como embarazos a término temprano 200 (39,8\%), a término completo 254 (50,6\%) y a término tardío 48 (9,6\%).

En relación con las características de la madre se encontró que la mediana de edad de las gestantes se comportó de manera similar (valor p = 0,0932) en los tres grupos (término completo, término temprano y término tardío). Se presentaron diferencias en el número de consultas prenatales de la madre, siendo el grupo de gestantes con nacimientos a término temprano las que accedieron a un menor número de consultas prenatales. La vía del parto y los días de hospitalización de la madre no mostraron diferencias entre los tres grupos. Se encontró mayor frecuencia de afiliación al régimen contributivo y de embarazos de alto riesgo en las gestantes a término temprano $(p=0,0074$ y $p=0,0001$, respectivamente).

En relación con las variables relacionadas con la hospitalización del recién nacido se encontraron diferencias estadísticamente significativas entre los grupos de análisis (valor $\mathrm{p}=0,0301$ ) (tabla 1 ). 


\begin{tabular}{|c|c|c|c|c|c|c|c|c|}
\hline \multicolumn{9}{|c|}{$\begin{array}{l}\text { Tabla } 1 . \\
\text { Características de la madre en gestaciones "normales" de } 37 \text { a } 41 \text { semanas en la } \\
\text { Clínica Universitaria Rafael Uribe Uribe de Cali (Colombia), } 2013\end{array}$} \\
\hline \multirow{3}{*}{ Característica } & \multicolumn{6}{|c|}{ Definición de embarazo } & \multirow{3}{*}{ Test } & \multirow{3}{*}{ Valor $\mathbf{p}$} \\
\hline & \multirow{2}{*}{\multicolumn{2}{|c|}{$\begin{array}{l}\text { Temprano } \\
\mathbf{n}=200\end{array}$}} & \multirow{2}{*}{\multicolumn{2}{|c|}{$\begin{array}{l}\text { Completo } \\
\mathbf{n}=254\end{array}$}} & \multirow{2}{*}{\multicolumn{2}{|c|}{$\begin{array}{l}\text { Tardío } \\
\mathbf{n}=48\end{array}$}} & & \\
\hline & & & & & & & & \\
\hline Edad de la madre $†$ & 24 & $(20-30,5)$ & 23 & $(19-30)$ & 23 & $(19-28)$ & 4,75 & 0,0932 \\
\hline Número consultas prenatales de la madre $\dagger$ & 6 & $(5-8)$ & 7 & $(6-8)$ & 7 & $(5-9)$ & 2,16 & 0,4278 \\
\hline Días de hospitalización de la madre † & 2,5 & $(1,4-4,8)$ & 2,6 & $(1,6-5,5)$ & 2,5 & $(1,7-6,6)$ & 1,19 & 0,5506 \\
\hline \multicolumn{9}{|l|}{ Afiliación de la madre $\S$} \\
\hline Subsidiado & 158 & $79,0 \%$ & 235 & $92,5 \%$ & 44 & $91,7 \%$ & \multirow{2}{*}{9,81} & \multirow{2}{*}{0,0074} \\
\hline Contributivo & 42 & $21,0 \%$ & 19 & $7,5 \%$ & 4 & $8,3 \%$ & & \\
\hline \multicolumn{9}{|l|}{ Diagnóstico materno al ingreso $\S$} \\
\hline Embarazo normal & 165 & $82,5 \%$ & 244 & $96,1 \%$ & 47 & $97,9 \%$ & \multirow{2}{*}{20,65} & \multirow{2}{*}{$<0,0001$} \\
\hline Embarazo de alto riesgo & 35 & $17,5 \%$ & 10 & $3,9 \%$ & 1 & $2,1 \%$ & & \\
\hline \multicolumn{9}{|l|}{ Vía de finalización del embarazo $\S$} \\
\hline Espontáneo & 114 & $57,0 \%$ & 171 & $67,3 \%$ & 27 & $56,3 \%$ & \multirow{2}{*}{3,08} & \multirow{2}{*}{0,2140} \\
\hline Cesárea & 86 & $43,0 \%$ & 83 & $32,7 \%$ & 21 & $43,8 \%$ & & \\
\hline \multicolumn{9}{|c|}{ Relacionados con la hospitalización del recién nacido $§$} \\
\hline Sífilis congénita & 7 & $3,5 \%$ & 9 & $3,5 \%$ & 2 & $4,2 \%$ & \multirow{5}{*}{16,99} & \multirow{5}{*}{0,0301} \\
\hline Trastornos hipertensivos madre & 8 & $4,0 \%$ & 4 & $1,6 \%$ & 0 & $0,0 \%$ & & \\
\hline Malformación & 8 & $4,0 \%$ & 3 & $1,2 \%$ & 0 & $0,0 \%$ & & \\
\hline Toxoplasmosis congénita & 3 & $1,5 \%$ & 5 & $2,0 \%$ & 0 & $0,0 \%$ & & \\
\hline Madre diabética & 0 & $0,0 \%$ & 3 & $1,2 \%$ & 2 & $4,2 \%$ & & \\
\hline
\end{tabular}

Al evaluar los resultados metabólicos en los neonatos se observan menor talla y menor peso promedio en gramos al nacer y mayor frecuencia de bajo peso al nacer en el grupo a término temprano $(\mathrm{p}<0,0001)$. El peso al nacer presentó una diferencia significativa entre los grupos (promedios de peso al nacer en el grupo a término temprano 2986 g, a término completo 3332,7 g y a término tardío $3462,7 \mathrm{~g}$ ); respecto a la talla, aquellos con nacimiento a término temprano mostraron valores más bajos en la talla (primer cuartil de $48 \mathrm{~cm}$ ), mientras que los nacimientos a término completo tuvieron valores por encima de $50 \mathrm{~cm}$. En las variables recién nacido grande, ictericia e hipoglicemia no se evidenció un comportamiento diferencial en los grupos objeto de estudio. Al evaluar la categoría de adaptación del recién nacido y comparar las variables: dificultad respiratoria, infecciones y días de hospitalización, no se encontraron diferencias estadísticamente significativas; por el contrario, la asfixia perinatal 
mostró diferencias entre los grupos, a expensas de término tardío, con un porcentaje de 18,8\% (valor $\mathrm{p}=0,0074)$ (tabla 2).

\section{DISCUSIÓN}

El presente estudio comparó los resultados obtenidos en recién nacidos a término temprano, a término completo y a término tardío, según la clasificación propuesta por la ACOG, donde se destaca la frecuencia de edad gestacional a término temprano con un 39,8\%. Se encontró mayor frecuencia de bajo peso al nacer en el grupo a término temprano, y mayor frecuencia de asfixia perinatal en el grupo a término tardío. No encontramos diferencias en los resultados perinatales adversos.

En cuanto a la frecuencia del embarazo a término temprano nuestros resultados son mayores a los descritos por Salemi et al. en Florida, Estados Unidos, que en una cohorte de 675.302 partos describió una frecuencia del 33,2\% de partos a

\begin{tabular}{|c|c|c|c|c|c|c|c|c|}
\hline \multirow{3}{*}{ Característica } & \multicolumn{6}{|c|}{ Definición de embarazo } & \multirow{3}{*}{ Test } & \multirow{3}{*}{ Valor $\mathbf{p}$} \\
\hline & \multirow{2}{*}{\multicolumn{2}{|c|}{$\begin{array}{l}\text { Temprano } \\
n=200\end{array}$}} & \multirow{2}{*}{\multicolumn{2}{|c|}{$\begin{array}{l}\text { Completo } \\
\mathbf{n}=254\end{array}$}} & \multirow{2}{*}{\multicolumn{2}{|c|}{$\begin{array}{l}\text { Tardío } \\
\mathrm{n}=48\end{array}$}} & & \\
\hline & & & & & & & & \\
\hline \multicolumn{9}{|l|}{ Metabólicas } \\
\hline Talla del nacido vivo $†$ & 50 & $(48-51)$ & 50 & $(49-52)$ & 51 & $(50-52)$ & 2,54 & 0,3866 \\
\hline Peso en gramos del menor al nacimiento $*$ & 2986 & 480,1 & 3332,7 & 475,7 & 3462,7 & 372,3 & 38,50 & $<0,0001$ \\
\hline Con BPN (peso < $2500 \mathrm{~g}) \S$ & 35 & $17,5 \%$ & 10 & $3,9 \%$ & 1 & $2,1 \%$ & 18,74 & 0,0001 \\
\hline RN Grande $\S$ & 1 & $0,5 \%$ & 2 & $0,8 \%$ & 2 & $4,2 \%$ & 3,06 & 0,2164 \\
\hline Ictericia $\S$ & 36 & $18,0 \%$ & 60 & $23,6 \%$ & 12 & $25,0 \%$ & 1,65 & 0,4376 \\
\hline Otras hipoglicemias $\S$ & 4 & $2,0 \%$ & 5 & $2,0 \%$ & 2 & $4,2 \%$ & 1,03 & 0,5983 \\
\hline \multicolumn{9}{|l|}{ Adaptativas } \\
\hline Dificultad respiratoria $\S$ & 22 & $11,0 \%$ & 20 & $7,9 \%$ & 2 & $4,2 \%$ & 3,48 & 0,1751 \\
\hline Asfixia perinatal $\S$ & 13 & $6,5 \%$ & 18 & $7,1 \%$ & 9 & $18,8 \%$ & 9,81 & 0,0074 \\
\hline Aspiración de meconio $\S$ & 1 & $0,5 \%$ & 3 & $1,2 \%$ & 1 & $2,1 \%$ & 0,51 & 0,7762 \\
\hline Otros $\S$ & 16 & $8,0 \%$ & 12 & $4,7 \%$ & 1 & $2,1 \%$ & 3,79 & 0,1504 \\
\hline APGAR $1 \dagger$ & 9 & $(8-9)$ & 9 & $(8-9)$ & 9 & $(6,7-9)$ & 2,89 & 0,2363 \\
\hline APGAR $2 \dagger$ & 10 & $(9-10)$ & 10 & $(9-10)$ & 10 & $(9-10)$ & 4,37 & 0,1127 \\
\hline \multicolumn{9}{|l|}{ Infecciosas $\S$} \\
\hline Sepsis & 62 & $31,0 \%$ & 95 & $37,4 \%$ & 13 & $27,1 \%$ & 2,34 & 0,3101 \\
\hline Corioamnionitis & 3 & $1,5 \%$ & 3 & $1,2 \%$ & 0 & $0,0 \%$ & 2,02 & 0,3642 \\
\hline Días de hospitalización del bebé † & 3,6 & $(1,7-5,2)$ & 3,6 & $(2,6-4,8)$ & 3,5 & $(1,8-5,1)$ & 1,31 & 0,5199 \\
\hline
\end{tabular}

$\S$ frecuencia (\%) - Prueba Z

* Media \pm DE, ANOVA - Prueba F

$\dagger$ Mediana (RIC), Kruskal-Wallis 
término temprano (8); los descritos por Furzan en Venezuela (9), en una cohorte de 2144 recién nacidos a término, quien encuentra un $24 \%$ del grupo a término temprano; de Ulubaş-Işık en Turquía (10), que informa una frecuencias de $28,5 \%$ en una cohorte retrospectiva de 31.170 partos, y de lo reportado por Sengupta en New York, Estados unidos (11), que informa un $27 \%$ en una cohorte retrospectiva de 33.478 partos, datos consistentes con el reporte de estadísticas vitales de Estados Unidos que registra una tendencia a la disminución, con un cambio en 2006 de 28,9 a 26,9\% en 2010, manteniendo un comportamiento similar a la fecha (12). Se destaca la elevada tasa de cesáreas presentes en los diferentes grupos a término temprano 43,0\%, término completo 32,7 \% y término tardío 43,8\%, sin presentar diferencias estadísticamente significativas, considerada muy superior a las metas definidas por la Organización Mundial de la Salud (OMS), que indica que esta debe oscilar entre un 10 y $15 \%$ (13). La cesárea per se parece ser un factor que empobrece el pronóstico en el grupo a término temprano $(8,11)$.

Nuestros hallazgos respecto a la mayor frecuencia de bajo peso al nacer es posible que estén asociados con la mayor presencia de embarazo de alto riesgo (17\%) en el grupo de embarazo a término temprano, lo que explica también la mayor frecuencia de hospitalización del neonato en este grupo. Este hallazgo también es descrito por Ulubaş-Işık en Turquía (10).

Nuestros resultados respecto a que no hay diferencias en la presencia de síndrome de dificultad respiratoria son similares a los informados por Salemi (8) en recién nacidos a término temprano nacidos por vía vaginal; este autor encuentra que la mayor frecuencia de dificultad respiratoria en este grupo se presenta en aquellos nacidos por cesárea. Por otra parte, Wang et al. (3), Furzan (9) UlubaşIşık (10), Sengupta (11) y Gómez-Pizarro (14) informan mayor frecuencia de dificultad respiratoria y admisión a UCI en los recién nacidos término temprano. Estos últimos autores también informan mayor frecuencia de sepsis neonatal, hipoglicemia, ictericia y necesidad de líquidos intravenosos, hallazgos que no coinciden con nuestros resultados. Respecto a la mayor frecuencia de asfixia perinatal en los fetos a término tardío, no encontramos referencias que soportaran este hallazgo.

Como limitaciones del estudio tenemos que se incluyó un $14 \%$ de pacientes con patologías maternas que fueron más frecuentes en el grupo a término temprano, y el pequeño tamaño de la muestra de este estudio que limita el poder para encontrar diferencias ya que se trató de un análisis exploratorio de los resultados neonatales. Sin embargo, los resultados son importantes ya que la clasificación es aplicable y deja varias preguntas que deberán ser contestadas en estudios regionales más grandes para evaluar la frecuencia de resultados perinatales adversos en el embarazo a término temprano según la vía de parto, y ajustando por factores maternos que afectan el metabolismo y la adaptación neonatal y su posible asociación con resultados adversos perinatales.

\section{CONCLUSIÓN}

En este estudio se analizaron 502 recién nacidos a término, de los cuales el 50,6\% fueron a término completo, seguidos del 39,8\% a término temprano, y 9,6\% a término tardío. No se encontraron resultados neonatales adversos más frecuentes en el grupo a término temprano. Se encontró mayor frecuencia de asfixia neonatal en el grupo a término tardío. Se requieren más estudios regionales para evaluar el valor pronóstico de esta clasificación propuesta en los tres grupos en relación con las variables de peso en gramos del menor al nacimiento y bajo peso al nacer.

\section{REFERENCIAS}

1. World Health Organization. International statistical classification of diseases and related health problems [Internet]. Vol. 2. WHO; 2004. http://apps.who.int/ iris/bitstream/10665/42980/1/9241546530_eng.pdf

2. Spong CY. Defining "term” pregnancy: recommendations from the defining "term" pregnancy workgroup. JAMA. 2013;309:2445-6. doi: 10.1001/ jama.2013.6235. 
3. Wang ML, Dorer DJ, Fleming MP, Catlin EA. Clinical outcomes of near-term infants. Pediatrics. 2004;114:372-6.

4. Tita AT, Landon MB, Spong CY, Lai Y, Leveno KJ, Varner MW, et al. Timing of elective repeat cesarean delivery at term and neonatal outcomes. N Engl J Med. 2009;360:111-20.

5. Definición de embarazo a término. Rev Obstet Ginecol Venez. 2013;73:285-6.

6. Reddy UM, Bettegowda VR, Dias T, YamadaKushnir T, Ko CW, Willinger M. Term pregnancy: a period of heterogeneous risk for infant mortality. Obstet Gynecol. 2011;117:1279-87. doi: 10.1097/ AOG.0b013e3182179e28.

7. Ehrenthal DB, Hoffman MK, Jiang X, Ostrum G. Neonatal outcomes after implementation of guidelines limiting elective delivery before 39 weeks of gestation. Obstet Gynecol. 2011;118:1047-55. doi: 10.1097/ AOG.0b013e3182319c58.

8. Salemi JL, Pathak EB, Salihu HM. Infant outcomes after elective early-term delivery compared with expectant management. Obstet Gynecol. 2016;127:657-6.

9. Furzán JA, Arteaga N, Luchón C, Expósito M, Henríquez A. Incidencia y morbilidad perinatal. Arch Venez Pueric Pediatría. 2012;75:108-12.
10. Ulubaş-Işık D, Erol S, Demirel N, Kale Y, Çelik İH, Tapısız ÖL, et al. Early-term delivery and adverse neonatal outcomes at a tertiary center in Turkey. Turk J Pediatr. 2015;57:547-52.

11. Sengupta S, Carrion V, Shelton J, Wynn RJ, Ryan RM, Singhal K, et al. Adverse neonatal outcomes associated with early-term birth. JAMA Pediatr. 2013;167:10539. doi: 10.1001/jamapediatrics.2013.2581.

12. Hoyert DL, Xu J. Deaths: Preliminary Data for 2011. National Vital Statistics Reports. Cent Dis Control Prev. 2012 [visitado 2016 Oct 23]; 61(1):53. Disponible en: http://large.stanford.edu/courses/2014/ph240/ poplawski2/docs/nvsr61_06.pdf

13. OMS. Declaración de la OMS sobre tasas de cesárea. Organización Mundial de la Salud. 2015 [visitado 2016 Oct 23]; 1-8. Disponible en: http://apps.who.int/iris/ bitstream/10665/161444/1/WHO_RHR_15.02_spa.pdf

14. Gómez-Pizarro CI, Rosas-Coronado MM, LeonelRivadeneyra S, Rojo-Quiñónez AR. Resultados neonatales en embarazo pretérmino tardío, término temprano y término completo. Bol Clínico Hosp Infant Estado Sonora. 2015;32:69-75. 